Article

\title{
The Effect of Thermal Radiation on Entropy Generation Due to Micro-Polar Fluid Flow Along a Wavy Surface
}

\author{
Cha'o-Kuang Chen, Yue-Tzu Yang and Kuei-Hao Chang * \\ Department of Mechanical Engineering, National Cheng-Kung University, Tainan 701, Taiwan; \\ E-Mails: ckchen@mail.ncku.edu.tw (C.-K.C.); ytyang@mail.ncku.edu.tw (Y.-T.Y.) \\ * Author to whom correspondence should be addressed; E-Mail: gtmpad@gmail.com; \\ Tel: $+886-6-275-7575$ ext. 62245 .
}

Received: 3 August 2011; in revised form: 29 August 2011 / Accepted: 31 August 2011 /

Published: 2 September 2011

\begin{abstract}
In this study, the effect of thermal radiation on micro-polar fluid flow over a wavy surface is studied. The optically thick limit approximation for the radiation flux is assumed. Prandtl's transposition theorem is used to stretch the ordinary coordinate system in certain directions. The wavy surface can be transferred into a calculable plane coordinate system. The governing equations of micro-polar fluid along a wavy surface are derived from the complete Navier-Stokes equations. A simple transformation is proposed to transform the governing equations into boundary layer equations so they can be solved numerically by the cubic spline collocation method. A modified form for the entropy generation equation is derived. Effects of thermal radiation on the temperature and the vortex viscosity parameter and the effects of the wavy surface on the velocity are all included in the modified entropy generation equation.
\end{abstract}

Keywords: entropy generation; thermal radiation; micropolar fluid; irreversibility; Bejan number; surface wavy; cubic spline

PACS Codes: 05.70.Ln 


\section{Nomenclature}

$\begin{array}{ll}a & \text { Amplitude of wavy surface } \\ B & \text { Dimensionless material parameter } \\ B e & \text { Bejan number } \\ B r & \text { Brinkman number } \\ C_{f} & \text { Skin-friction coefficient } \\ h & \text { Heat transfer coefficient } \\ j & \text { Microinertia density } \\ K_{f} & \text { Thermal Conductivity } \\ L & \text { Characteristic length } \\ N & \text { Dimensionless microrotation } \\ N_{F} & \text { Entropy generation due to fluid friction } \\ N_{H} & \text { Entropy generation due to heat transfer } \\ N_{S} & \text { Entropy generation number } \\ N_{R} & \text { Radiation-conduction parameter } \\ P & \text { Pressure } \\ \operatorname{Pr} & \text { Prandtl number } \\ R & \text { Vortex viscosity parameter } \\ q_{r} & \text { Radiation heat flux } \\ \operatorname{Re} & \text { Reynolds number } \\ S(x) & \text { Surface geometry function } \\ U_{w} & \text { x component of velocity of the inviscid flow evaluated at surface } \\ u & \text { Dimensionless x component of velocity } \\ T & \text { Temperature } \\ v & \text { Dimensionless y component of velocity } \\ x, y & \text { Axial and transverse (Cartesian) coordinates, respectively } \\ & \end{array}$

Greek Symbols

$\begin{array}{ll}\alpha & \text { Wavy amplitude-wavelength ratio } \\ \alpha_{r} & \text { The mean extinction coefficient } \\ \beta & \text { Thermal expansion coefficient of fluid } \\ \gamma & \text { Spin-gradient viscosity } \\ \theta & \text { Dimensionless temperature } \\ \kappa & \text { Vortex viscosity } \\ \lambda & \text { Material parameter } \\ \mu & \text { Dynamic viscosity } \\ \nu & \text { Kinematic viscosity } \\ \rho & \text { Density of fluid } \\ \Omega & \text { Dimensionless temperature difference }\end{array}$




\section{Subscripts}

$\begin{array}{ll}w & \text { Wall surface } \\ \infty & \text { Free stream condition }\end{array}$

Superscripts

$\begin{array}{ll}- & \text { Dimensional variables } \\ , & \text { Dimensionless quantity } \\ & \text { Derivative with respect to } \mathrm{x}\end{array}$

\section{Introduction}

In recent years, the heat convection of wavy surfaces has been studied extensively because of its wide practical applications. Yao $[1,2]$ proposed a simple transformation to study the natural convection heat transfer of isothermal vertical wavy surfaces, e.g., sinusoidal surfaces. Using transformation, the boundary layer equations of natural convection in Newtonian fluids can be solved by a numerical finite difference method. Results show that the local heat transfer rate varies periodically along the wavy surface, with a frequency equal to twice the frequency of the surface. Study of natural convection and study of mixed convection along a vertical wavy surface was provided by Moulic and Yao [3]. They showed that the total mixed convection heat flux along a wavy surface is smaller than that of a flat surface. Yao [4] showed that the enhanced total heat-transfer rate seems to depend on the ratio of the amplitude and wavelength of a surface. Wang and Chen [5] studied the rates of heat transfer for flow through a sinusoidally curved converging-diverging channel. Results showed that flow through a periodic array of wavy-wall channels forms a highly complex pattern which is composed of a strong forward flow and an oppositely directed recirculating flow with each wave. Micro-polar fluids possess certain microscopic effects that come from local structure and micro-motion of the fluid [6-9], and can be used to study the behavior in fluid media such as polymeric fluids, liquid crystals and animal blood. Wang and Chen [10-12] studied micro-polar fluids and heat convection, showing that the harmonic curves for the local skin friction coefficient and the local $\mathrm{Nu}$ have the same frequency as the frequency of the wavy surface. Moreover, the vortex viscosity parameter tends to decrease the heat transfer rate and to increase the skin friction coefficient. Additionally, literature regarding non-Newtonian fluids such as Lien et al. [13,14], Yang et al. [15], Chen et al. [16] and Wang [17], are available for different thermal conditions and field effects. The above studies show that the heat transfer of an irregular surface is a topic of fundamental importance and is encountered in heat transfer systems such as flat plate solar collectors, condensers in refrigerators and fins used to enhance the rate of heat transfer in electronic equipment cooling systems. Lien et al. [18] studied heat transfer in a plate fin, showing that the modified local heat transfer coefficient is determined by a highly coupled interaction among the fin conduction, radiation and fluid convection flow, assuming the optically thick limit approximation for radiation flux. The optically thick approximation for radiation flux was derived by Rosseland [19]. Many studies have used this approximation, e.g., Novotny and Yang [20] who discussed the role of the optically thick approximation in convection-radiation interaction situations. Chen and Ozisic [21] studied radiation with free convection in absorbing, emitting and scattering media. Hossain and 
Takhar [22] studied radiation effects on mixed flow along a heated vertical flat plate with the Rosseland diffusion approximation. Elsayed [23] studied thermal buoyancy and thermal radiation effects on the development of a boundary layer flow past a horizontal plate, with emphasis placed on energy conservation and efficient use of energy. Analysis of thermodynamic irreversibility appears to be increasingly important [24-28]. From an engineering viewpoint, thermodynamic irreversibility is particularly applicable in the analysis of complex thermal systems [29]. The analysis of thermodynamic irreversibility enables us to identify the irreversibility associated with various components and avoid loss of available power [30]. This information can be employed to design thermal systems, guide efforts to reduce sources of irreversibility in engineering systems, estimate the cost of engineering systems and optimize complex systems [31-35]. In view of the above, it is seen that wavy surface analysis in the literature tends to focus on the first law of thermodynamics. On the other hand, it is seen that thermodynamic irreversibility is also a topic of importance. This present work focuses on enhanced modeling of entropy generation due to micro-polar fluid flow along the wavy surface including radiation effect.

\section{Mathematical Formulation}

Consider a two dimensional semi-infinite wavy surface that is placed in a fluid field of temperature $=T_{\infty}$. The wavy surface and the outside free stream are parallel. Additionally, the surface-temperature is maintained as $T_{w}$. The formula of the wavy surface can be described as:

$$
\bar{y}=\bar{S}(\bar{x})=\bar{a} \sin ^{2}(\pi \bar{x} / L)
$$

In this formula, $\bar{a}$ is the amplitude of the wavy surface and $L$ is the length of a surface wave. Figure 1 illustrates the physical module and coordinate system.

Figure 1. Physical module and coordinates system.

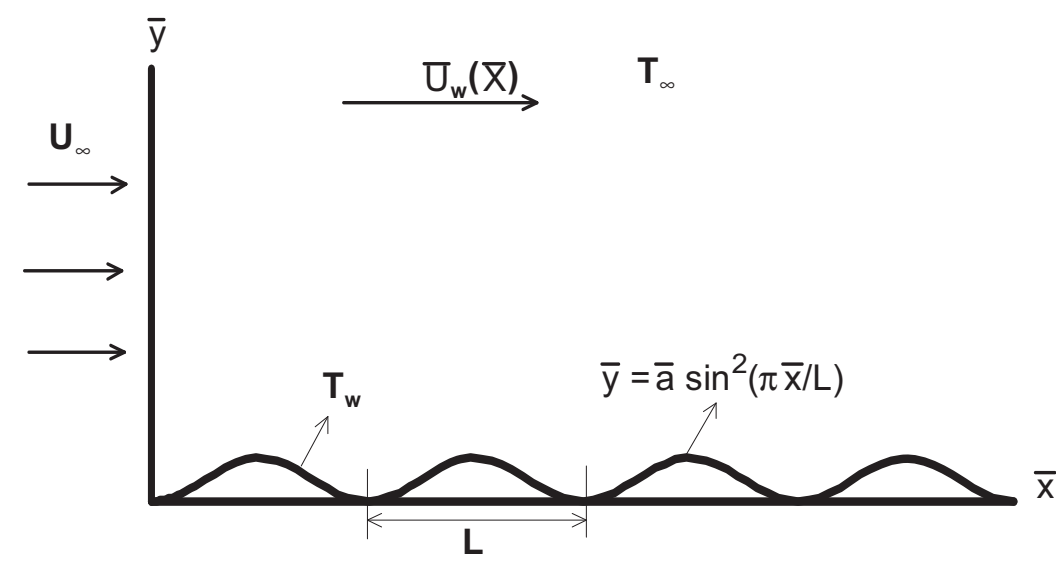

The governing equations for micro-polar parameter under consideration can be written as:

$$
\begin{gathered}
\frac{\partial \bar{u}}{\partial \bar{x}}+\frac{\partial \bar{v}}{\partial \bar{y}}=0 \\
\rho\left(\bar{u} \frac{\partial \bar{u}}{\partial \bar{x}}+\bar{v} \frac{\partial \bar{u}}{\partial \bar{y}}\right)=-\frac{\partial \bar{p}}{\partial \bar{x}}+(\mu+\kappa)\left(\frac{\partial^{2} \bar{u}}{\partial \bar{x}^{2}}+\frac{\partial^{2} \bar{u}}{\partial \bar{y}^{2}}\right)+\kappa \frac{\partial v_{3}}{\partial \bar{y}}
\end{gathered}
$$




$$
\begin{gathered}
\rho\left(\bar{u} \frac{\partial \bar{v}}{\partial \bar{x}}+\bar{v} \frac{\partial \bar{v}}{\partial \bar{y}}\right)=-\frac{\partial \bar{p}}{\partial \bar{y}}+(\mu+\kappa)\left(\frac{\partial^{2} \bar{v}}{\partial \bar{x}^{2}}+\frac{\partial^{2} \bar{v}}{\partial \bar{y}^{2}}\right)+\kappa\left(-\frac{\partial v_{3}}{\partial \bar{x}}\right) \\
\rho j\left(\bar{u} \frac{\partial v_{3}}{\partial \bar{x}}+\bar{v} \frac{\partial v_{3}}{\partial \bar{y}}\right)=\kappa\left(\frac{\partial \bar{v}}{\partial \bar{x}}-\frac{\partial \bar{u}}{\partial \bar{y}}-2 v_{3}\right)+\gamma\left(\frac{\partial^{2} v_{3}}{\partial \bar{x}^{2}}+\frac{\partial^{2} v_{3}}{\partial \bar{y}^{2}}\right) \\
\bar{u} \frac{\partial T}{\partial \bar{x}}+\bar{v} \frac{\partial T}{\partial \bar{y}}=\frac{K_{f}}{\rho C_{p}}\left(\frac{\partial^{2} T}{\partial \bar{x}^{2}}+\frac{\partial^{2} T}{\partial \bar{y}^{2}}\right)-\frac{1}{\rho C_{p}}\left(\frac{\partial q_{r}}{\partial \bar{x}}+\frac{\partial q_{r}}{\partial \bar{y}}\right)
\end{gathered}
$$

The optically thicken limit approximation for the radiation flux is:

$$
q_{r}=-\left(\frac{4 \sigma}{3 K_{f} \alpha_{r}}\right) \nabla T^{4}
$$

where $\sigma$ is the Stefan-Boltzman constant, $\alpha_{r}$ is the extinction coefficient, $q_{r}$ is the radiation heat flux. $v_{3}$ is the micro-rotation component and $C_{P}$ is the specific heat of the fluid at constant. Now the dimensionless variables are defined as:

$$
\begin{aligned}
& \tilde{x}=\frac{\bar{x}}{L} \quad \tilde{y}=\frac{\bar{y}}{L} \quad \alpha=\frac{\bar{a}}{L} \quad \tilde{u}=\frac{\bar{u}}{U_{\infty}} \quad \tilde{v}=\frac{\bar{v}}{U_{\infty}} \quad U_{w}=\frac{\bar{U}_{w}}{U_{\infty}} \\
& \theta=\frac{T-T_{\infty}}{T_{w}-T_{\infty}} \quad S(\tilde{x})=\frac{\bar{S}(\bar{x})}{L} \quad \tilde{p}=\frac{\bar{p}}{\rho U_{\infty}^{2}} \quad \operatorname{Re}=\frac{\rho U_{\infty} L}{\mu} \quad \operatorname{Pr}=\frac{\mu C_{p}}{K_{f}} \\
& N_{R}=\frac{4 \sigma T_{\infty}^{3}}{K_{f} \alpha_{r}} \quad \tilde{N}=\frac{v_{3} L}{U_{\infty}} \quad B=\frac{L \mu}{\rho j U_{\infty}} \quad \lambda=\frac{\gamma}{j \mu} \quad R=\frac{\kappa}{\mu} \quad \theta_{w}=\frac{T_{w}}{T_{\infty}}
\end{aligned}
$$

The transformed variables are:

$$
\begin{aligned}
& \hat{x}=\tilde{x} \quad \hat{y}=(\tilde{y}-S(\tilde{x})) \operatorname{Re}^{\frac{1}{2}} \quad \hat{u}=\tilde{u} \quad \hat{v}=\left(\tilde{v}-S(\tilde{u}) \operatorname{Re}^{\frac{1}{2}}\right. \\
& \hat{p}=\tilde{p}-\widetilde{p}_{\infty} \hat{N}=\widetilde{N} \operatorname{Re}^{\frac{-1}{2}}
\end{aligned}
$$

By substituting the dimensionless and transformed variables into Equations (2)-(5) and then transforming the wavy surface into flat surface by Prandtl's transposition theorem [2], then letting $\mathrm{Re} \rightarrow \infty$ (boundary layer approximation), the transformed equations can be given as:

$$
\begin{gathered}
\frac{\partial \hat{u}}{\partial \hat{x}}+\frac{\partial \hat{v}}{\partial \hat{y}}=0 \\
\hat{u} \frac{\partial \hat{u}}{\partial \hat{x}}+\hat{v} \frac{\partial \hat{u}}{\partial \hat{y}}=-\frac{\partial \hat{p}}{\partial \hat{x}}+\operatorname{Re}^{1 / 2} S^{\prime} \frac{\partial \hat{p}}{\partial \hat{y}}+\left(1+S^{\prime 2}\right)(1+R) \frac{\partial^{2} \hat{u}}{\partial \hat{y}^{2}}+R \frac{\partial \hat{N}}{\partial \hat{y}} \\
\hat{u}^{2} S^{\prime \prime}=S^{\prime} \frac{\partial \hat{p}}{\partial \hat{x}}-\left(1+S^{\prime 2}\right) \operatorname{Re}^{1 / 2} \frac{\partial \hat{p}}{\partial \hat{y}} \\
\hat{u} \frac{\partial \hat{N}}{\partial \hat{x}}+\hat{v} \frac{\partial \hat{N}}{\partial \hat{y}}=R B\left(-\left(1+S^{\prime 2}\right) \frac{\partial \hat{u}}{\partial \hat{y}}-2 \hat{N}\right)+\lambda\left(1+S^{\prime}\right) \frac{\partial^{2} N}{\partial \bar{y}^{2}} \\
\hat{u} \frac{\partial \theta}{\partial \hat{x}}+\hat{v} \frac{\partial \theta}{\partial \hat{y}}=\frac{1+S^{\prime 2}}{\operatorname{Pr}}\left[\frac{4}{3} N_{R}\left(1+\left(\theta_{w}-1\right) \theta\right)^{3}\right] \frac{\partial^{2} \theta}{\partial \hat{y}^{2}}
\end{gathered}
$$


Equation (9) indicates that the pressure gradient along the $y$-direction is $\mathrm{O}\left(\mathrm{Re}^{-1 / 2}\right)$, which implies that the lowest-order pressure gradient along the $x$-direction can be determined from the inviscid flow solution, that is:

$$
\frac{\partial \hat{p}}{\partial \hat{x}}=-\left[\left(1+S^{2}\right) U_{w} U_{w}^{\prime}+S^{\prime} S^{\prime \prime} U_{w}^{2}\right]
$$

Eliminating $\partial \hat{p} / \partial \hat{y}$ between Equations (8) and (9) and using Equation (11) gives:

$$
\hat{u} \frac{\partial \hat{u}}{\partial \hat{x}}+\hat{v} \frac{\partial \hat{u}}{\partial \hat{y}}=\frac{1}{\left(1+S^{\prime 2}\right)}\left(-\frac{\partial \hat{p}}{\partial \hat{x}}-\hat{u}^{2} S^{\prime} S^{\prime \prime}\right)+\left(1+S^{\prime 2}\right) \frac{\partial^{2} \hat{u}}{\partial \hat{y}^{2}}
$$

with the $x, y, u, v$ and $N$ variables defined as:

$$
\begin{aligned}
& x=\hat{x} \quad y=\hat{y}\left(2 \hat{x} / U_{w}\right)^{\frac{-1}{2}} \\
& u=\hat{u} / U_{w} \quad v=\hat{v}\left(2 \hat{x} / U_{w}\right)^{\frac{1}{2}} \quad N=\hat{N}\left(2 \hat{x} / U_{w}\right)^{\frac{1}{2}}
\end{aligned}
$$

Then Equations (7), (12), (10a) and (10b) can be transformed into, respectively:

$$
\begin{gathered}
2 x \frac{\partial u}{\partial x}-y\left(1-\frac{x U_{w}^{\prime}}{U_{w}}\right)\left(\frac{\partial u}{\partial y}\right)+\frac{\partial v}{\partial y}+2 x u \frac{U_{w}^{\prime}}{U_{w}}=0 \\
2 x u \frac{\partial u}{\partial x}+\left[v-y u\left(1-x \frac{U_{w}^{\prime}}{U_{w}}\right)\right] \frac{\partial u}{\partial y}+2 x\left(u^{2}-1\right)\left(\frac{S^{\prime} S^{\prime \prime}}{1+S^{\prime 2}}+\frac{U_{w}^{\prime}}{U_{w}}\right) \\
=(1+R)\left(1+S^{\prime 2}\right) \frac{\partial^{2} u}{\partial y^{2}}+\frac{R}{U_{w}} \frac{\partial N}{\partial y} \\
2 x u \frac{\partial N}{\partial x}+\left[v-y u\left(1-x \frac{U_{w}^{\prime}}{U_{w}}\right)\right] \frac{\partial N}{\partial y}-u N\left(1-x \frac{U_{w}^{\prime}}{U_{w}}\right) \\
=x R B\left[-2\left(1+S^{\prime 2}\right) \frac{\partial u}{\partial y}-4 \frac{N}{U_{w}}\right]+\lambda\left(1+S^{\prime 2}\right) \frac{\partial^{2} N}{\partial y^{2}} \\
2 x \theta \frac{\partial \theta}{\partial x}+\left[v-y u\left(1-x \frac{U_{w}^{\prime}}{U_{w}}\right)\right] \frac{\partial \theta}{\partial y}=\frac{1+S^{\prime 2}}{\operatorname{Pr}}\left[1+\frac{4}{3} N_{R}\left(1+\left(\theta_{w}-1\right) \theta\right)^{3}\right] \frac{\partial^{2} \theta}{\partial y^{2}}
\end{gathered}
$$

The corresponding boundary conditions are:

$$
\begin{array}{lrll}
y=0: & \theta=1 & u=v=0 & N=0 \\
y \rightarrow \infty: & \theta \rightarrow 0 & u \rightarrow 1 & N \rightarrow 0
\end{array}
$$

Next the inviscid flow along the wavy surface is obtained. The inviscid solution here is valid only for small values of the amplitude-wave length ratio. The potential-flow solution $U_{w}(x)$ for small values of $\alpha(<<1)$ was reported by Moulic et al. [36]. $U_{w}(x)$ can be expressed as:

$$
U_{w}(x)=1+\frac{1}{\pi} \int_{0}^{\infty} \frac{S^{\prime}(t)}{x-t} d t+O\left(\alpha^{2}\right)
$$

Removing the singular point in the integral by the residue theorem, Equation (15a) can be written as: 


$$
U_{w}(x)=1+\alpha\left[-\pi \cos (2 \pi x)+\int_{0}^{\infty} \frac{\sin (2 \pi t)}{x+t} d t\right]+O\left(\alpha^{2}\right)
$$

For a two-dimensional Cartesian system, the local entropy generation rate is:

$$
\begin{aligned}
& S_{\text {gen }}^{\prime \prime \prime}=\frac{K_{f}}{T^{2}}\left[\left(\frac{\partial T}{\partial x}\right)^{2}+\left(\frac{\partial T}{\partial y}\right)^{2}+N_{R}\left(\frac{\partial T^{4}}{\partial x}+\frac{\partial T^{4}}{\partial y}\right)\left(\frac{\partial T}{\partial x}+\frac{\partial T}{\partial y}\right)\right] \\
& +\frac{\mu+\kappa}{T}\left\{2\left[\left(\frac{\partial u}{\partial x}\right)^{2}+\left(\frac{\partial v}{\partial y}\right)^{2}\right]+\left(\frac{\partial u}{\partial y}+\frac{\partial v}{\partial x}\right)^{2}\right\}
\end{aligned}
$$

According to Bejan [37,38], the dimensionless entropy generation equation is the entropy generation number which is the ratio of the volumetric entropy generation rate to the characteristic entropy generation rate. Thus, from Equation (16a), the entropy generation number can be expressed as:

$$
N_{s}=\left(1+\frac{4}{3} N_{R} \theta_{w}^{3}\right)\left[U_{w}\left(1+S^{\prime 2}\right)\left(\frac{\partial \theta}{\partial y}\right)^{2}\right]+\frac{B r}{\Omega}(1+R)\left[U_{w}^{3}\left(1-S^{\prime 2}\right)^{2}\left(\frac{\partial u}{\partial y}\right)^{2}\right]=N_{H}+\frac{B r}{\Omega}(1+R) N_{F}
$$

where the characteristic entropy generation rate is $K_{f}\left(\Delta T / T_{\infty} L\right)^{2}, N_{H}$ is the heat transfer irreversibility, $N_{F}$ is the fluid friction irreversibility and $N_{F} / N_{H}$ is the irreversible distribution rate $(\varphi)$ presented by Bejan. Thus, the Bejan number can be written as [28,39]:

$$
B e=\frac{1}{1+\varphi}
$$

The Bejan number has a value between 0 and 1 . If the Bejan number equals 0 , then irreversibility is dominated by fluid friction. If the Bejan number equals 1 , then irreversibility is dominated by heat transfer. Friction and heat transfer irreversibility are the same when the Bejan number equals 0.5.

\section{Numerical Method}

An improved version of the cubic spline collocation method [40] is used to perform numerical computation in this study [41]. Using cubic spline collocation, Equations (14a)-(14d) can be written as:

$$
\Phi_{i, j}^{n+1}=F_{i, j}+G_{i, j} m_{f_{i, j}}^{n+1}+S_{i, j} M_{f_{i, j}}^{n+1}
$$

where the values $F, G$ and $S$ are known coefficients evaluated at previous time steps, $\mathrm{i}$ and $\mathrm{j}$ are computational nodes, $\mathrm{n}$ refers to the time step, $\Phi$ represents $\mathrm{u}$ and $\theta$ are as shown in Table 1 . When combined with cubic spline relations, Equation (17a) can be written as:

$$
A_{i, j} \psi_{i, j-1}+B_{i, j} \psi_{i, j}+C_{i, j} \psi_{i, j+1}=D_{i, j}
$$

The Thomas algorithm is then used to solve Equation (17b). 
Table 1. The value of $F_{i, j}, G_{i, j}$, and $S_{i, j}$.

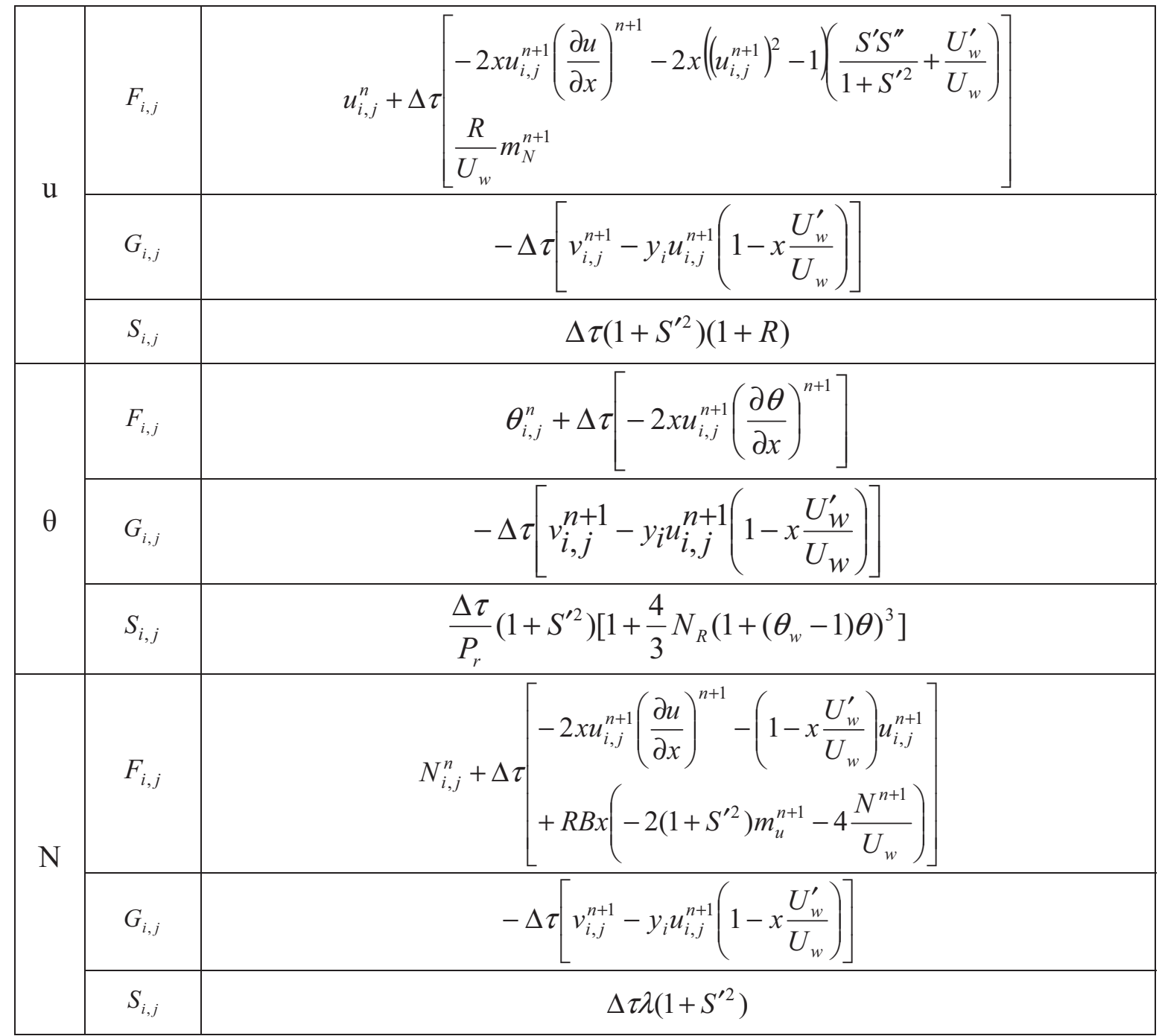

\section{Results and Discussion}

An accuracy test of grid fineness is made for grids of $100 \times 20,100 \times 50,100 \times 150,25 \times 50$, $50 \times 50$ and $100 \times 50$. Results are listed in Table 2 . In this study we use a $100 \times 50$ nonuniform grid with smaller spacing of the mesh points in the neighborhood of the fluid-solid boundary in the $y$ direction. In order to verify the accuracy of the solution, numerical results are obtained for Newtonian fluid flow over a flat plate $(\alpha=0)$. The $\mathrm{N}_{\mathrm{H}}$ and $\mathrm{N}_{\mathrm{F}}$ results from Equation (16b) are found to be in good agreement with results of previous study $[42,43]$. Although Figure 2, does not include any variation, it is included in order to verify the accuracy of the solution, since it demonstrates good agreement with results from the Chen et al. [43]. In addition, Figure 2 shows that the Bejan number for a flat plane is 0.5 , which means that both of heat transfer and flow friction contribution to entropy generation are in the same level. 
Table 2. Local heat transfer rate and local skin friction coefficient for different grids.

\begin{tabular}{|c|c|c|c|c|}
\hline \multirow{2}{*}{} & $\left(2 / \mathrm{Re}_{\bar{x}}\right)^{1 / 2} N u_{\bar{x}}$ & \multicolumn{2}{c|}{$\left(2 \mathrm{Re}_{\bar{x}}\right)^{1 / 2} C_{f}$} \\
\cline { 2 - 5 } & $\mathrm{x}=0.16$ & $\mathrm{x}=4$ & $\mathrm{x}=0.16$ & $\mathrm{x}=4$ \\
\hline \multicolumn{3}{|c|}{ Steady state solutions: $\mathrm{Pr}=0.73, \alpha=0.002, x \in[0,4], y \in[0,10]$} \\
\hline $100 \times 20$ & 0.393842 & 0.379809 & 1.131646 & 0.968401 \\
\hline $100 \times 50$ & 0.394535 & 0.376537 & 1.131432 & 0.960356 \\
\hline $25 \times 50$ & 0.394550 & 0.375848 & 1.129922 & 0.958338 \\
\hline $50 \times 50$ & 0.394376 & 0.375799 & 1.132669 & 0.959618 \\
\hline $100 \times 50$ & 0.394393 & 0.376083 & 1.130753 & 0.956972 \\
\hline $100 \times 75^{(*)}$ & 0.391625 & 0.378118 & 1.123619 & 0.959037 \\
\hline $100 \times 200^{(*)}$ & 0.393746 & 0.376413 & 1.127222 & 0.959405 \\
\hline${ }^{(*)}:$ Uniform grid (y direction); & 0.394248 & 0.967 \\
\hline
\end{tabular}

Figure 2. Axial distribution of Bejan number for forced convection while $\alpha=0, \operatorname{Pr}=1$, $\mathrm{Br} / \Omega=1$ and $N_{R}=0$.

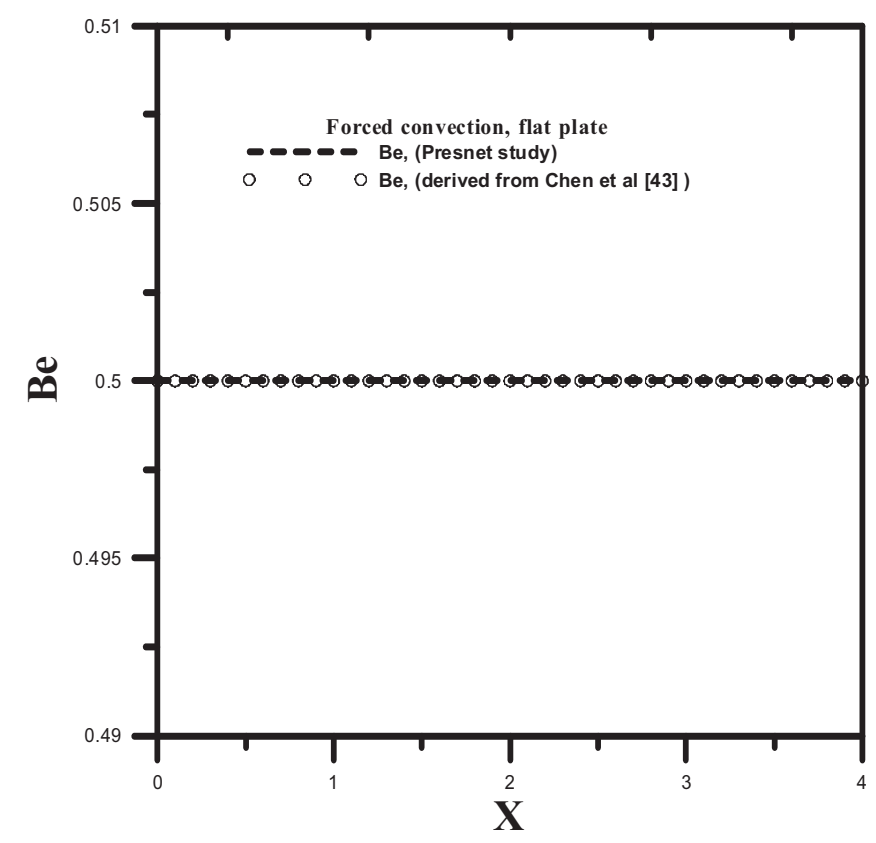

In order to solve Equations (14a)-(14d), Simpson's integral is applied to calculate the value of $U_{\mathrm{w}}(x)$ in Equation (15b). As shown in Figure 3, while ascending along the wavy surface (slope $S^{\prime}$ is positive from trough to crest), the flow accelerates and the pressure gradient is negative; while descending along the wavy surface (slope $S^{\prime}$ is negative from crest to trough), the flow decelerates and the pressure gradient is positive. Thus $U_{\mathrm{w}}(x)$ varies periodically along the surface with a frequency equal to that of the wavy surface and increases with increasing amplitude-wave length ratio. The pressure distribution has a frequency equal to that of the wavy surface. The maximum and minimum values of the pressure gradient occur at the points of inflection of the wavy surface, and the pressure gradient tends to increase as the amplitude-wave length ratio increases. 
Figure 3. Inviscid surface velocity distribution and axial distribution of pressure gradient.

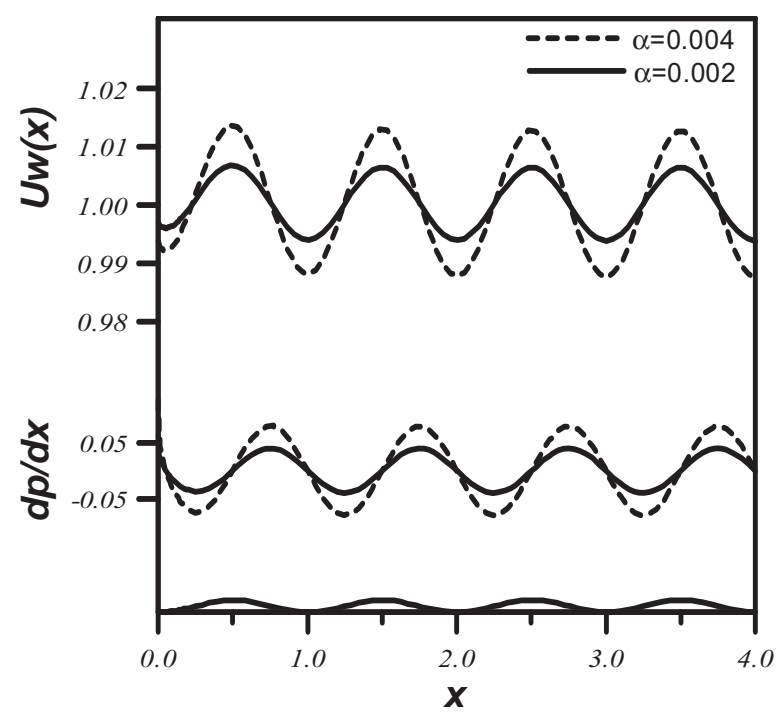

Referring to Figure 4, it is observed that the amplitude of $\mathrm{N}_{\mathrm{H}}$ increases with increasing $\mathrm{x}$. It is seen that increasing the value of $\mathrm{N}_{\mathrm{R}}$ leads to a shift of the $\mathrm{N}_{\mathrm{H}}$ curve upwards. The results indicate that increasing $\mathrm{N}_{\mathrm{R}}$ leads to increased temperature distribution, and also to enhanced entropy generation due to heat transfer of the flow field. Figure 5 shows that increasing $N_{F}$ has no effect with regard to increasing $\mathrm{N}_{\mathrm{R}}$. This is because the values of both $R$ and $\mathrm{N}_{\mathrm{R}}$ are not coupled and the value of $B r / \Omega$ is constant. In other words, when the value of $N_{R}$ is increases, the heat flux of thermal radiation is absorbed into the fluid. Although the temperature of the fluid is increases with increasing $\mathrm{N}_{R}$, phase change does not take place in the micro-polar fluid. In consequence, the total fluid viscosity value and the entropy generation value due to fluid friction are constant. Figure 5 shows that the value of $\mathrm{N}_{\mathrm{F}}$ in a micro-polar fluid tends to decrease rapidly near the leading edge as the fluid moves downstream, which is different than the behavior for Newtonian fluids. The behavior is the same as that observed by Wang [10] in his study of micro-polar fluids.

Figure 4. Axial distribution of $\mathrm{N}_{\mathrm{H}}$ for different $N_{R}$ while $\alpha=0.002, \operatorname{Pr}=0.7, B r / \Omega=1, \theta_{w}=1.2$.

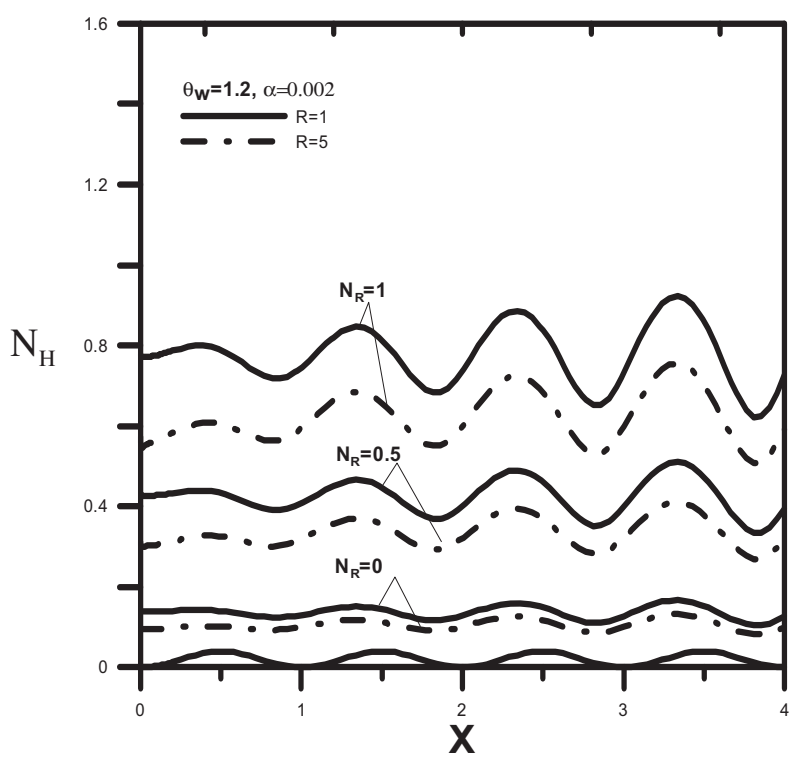


Figure 5. Axial distribution of $\mathrm{N}_{\mathrm{F}}$ for different $N_{R}$ while $\alpha=0.002, \operatorname{Pr}=0.7, B r / \Omega=1, \theta_{w}=1.2$.

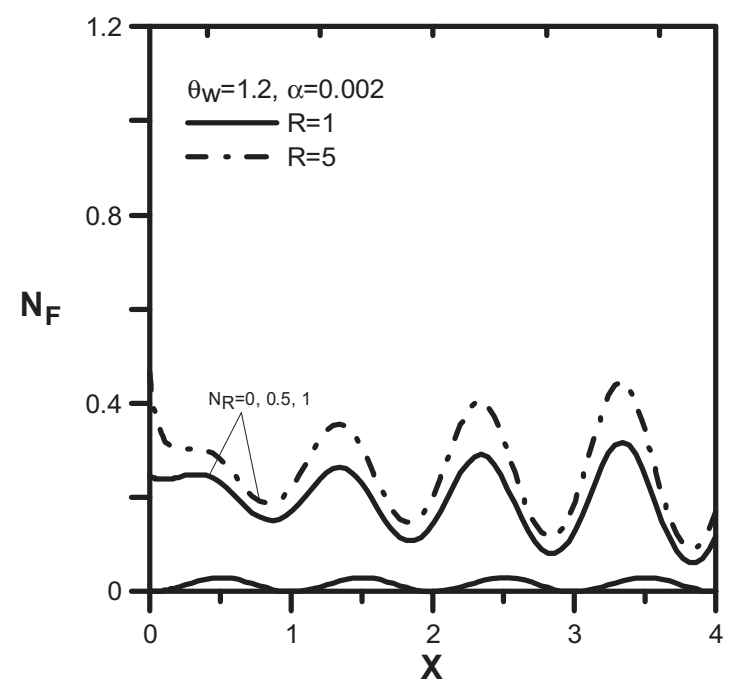

Figures 4 and 5 show that as the vortex viscosity parameter $R$ increases, $N_{H}$ decreases and $N_{F}$ increases. This is because an increase in the vortex viscosity results in an increase in the total viscosity of fluid flow, inducing increased $\mathrm{N}_{\mathrm{F}}$ due to fluid friction and decreased $\mathrm{N}_{\mathrm{H}}$ due to heat transfer. It is observed that the amplitude of $\mathrm{N}_{\mathrm{H}}$ and $\mathrm{N}_{\mathrm{F}}$ increase with increasing $\mathrm{x}$.

Figure 6 shows that when the value of $N_{R}$ equals zero, then the crests of the $N_{S}$ harmonics when $R$ equals 5 are larger than when $\mathrm{R}$ equals 1 , and the crests of the $\mathrm{N}_{\mathrm{S}}$ harmonics increase with an increasing values of $N_{R}$. It is also seen that when the value of $N_{R}$ equals 1 , then the crests of the $N_{S}$ harmonic when R equals 5 are smaller than when $\mathrm{R}$ equals 1 .

Figure 6. Axial distribution of $\mathrm{N}_{\mathrm{S}}$ for different $N_{R}$ while $\alpha=0.002, \operatorname{Pr}=0.7, B r / \Omega=1, \theta_{w}=1.2$.

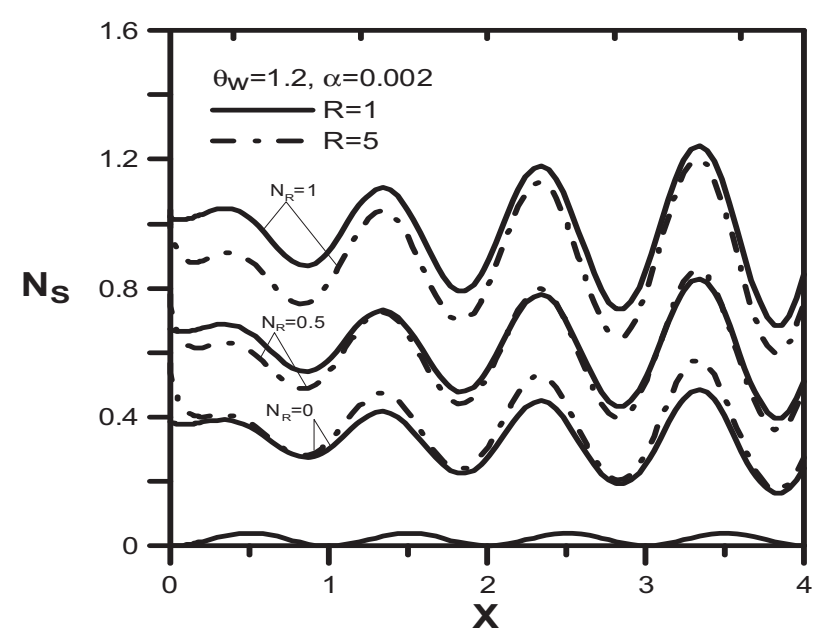

The reason for this behavior is that the $\mathrm{N}_{\mathrm{H}}$ harmonics trend to increase but the $\mathrm{N}_{\mathrm{F}}$ harmonics remain unchanged when $\mathrm{N}_{R}$ increases. It is observed that the amplitude of $\mathrm{N}_{\mathrm{S}}$ increases with increasing $\mathrm{x}$, which corresponds to the behavior of the harmonics of $\mathrm{N}_{\mathrm{H}}$ and $\mathrm{H}_{\mathrm{F}}$. This phenomenon can be understood from the fact that entropy in the system trends to increase with increasing $\mathrm{x}$. In addition, the harmonics of $\mathrm{N}_{\mathrm{H}}, \mathrm{N}_{\mathrm{F}}$ and $\mathrm{N}_{\mathrm{S}}$ show a periodic variation with a frequency equal to the frequency of the wavy surface, but their maximum and minimum values do not occur exactly at the crests and 
troughs of the wavy surface. Note that for a complete cycle $(1<\mathrm{x}<2)$, the maximum value occurs at $\mathrm{x}=1.35$, not at the crest of the wavy surface $(\mathrm{x}=1.5)$, and the minimum value occurs at $\mathrm{x}=1.85$, not at the trough of wavy $\operatorname{surface}(\mathrm{x}=2)$.

From Figure 7, in addition to the first crest of the Be harmonic, it is found that the crests of the Be harmonic are larger than 0.5 when $R$ equals 1 and $N_{R}$ equals 0 (no thermal radiation), which means that heat transfer dominates entropy generation at the crests of the wavy surface when when $R$ equals 1 . However, it is seen that the crests the of Be harmonic are smaller than 0.5 when $\mathrm{R}$ equals 5 and $\mathrm{N}_{\mathrm{R}}$ equals 0 (no thermal radiation). This indicates that friction dominates entropy generation in the crests of the wavy surface when $R$ equals 5 . In addition, the Be harmonics are larger than 0.5 when $\mathrm{N}_{R}$ is over 0.5 , which means that as the value of $\mathrm{N}_{\mathrm{R}}$ increases, the thermal radiation heat flux is absorbed into the fluid, which then leads to enhanced entropy generation due to heat transfer of the flow field, hence heat transfer dominates entropy generation over the wavy surface.

Figure 7. Axial distribution of Be number for different $N_{R}$ while $\alpha=0.002, \operatorname{Pr}=0.7$, $B r / \Omega=1, \theta_{w}=1.2$.

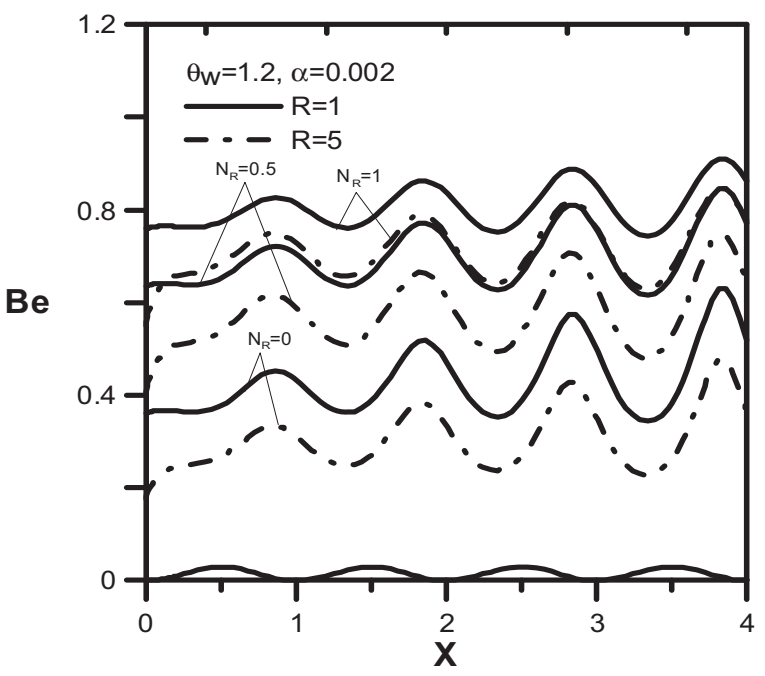

For a complete wave $(0.5<\mathrm{x}<1.5)$, as seen in Figure 8 , the temperature of fluid motion in a trough is higher than in a crest, and the temperature of fluid motion in the troughs and crests increases with increasing $\mathrm{N}_{\mathrm{R}}$. Figure 9 shows that increasing $\mathrm{N}_{\mathrm{R}}$ has no effect on the velocity of both crests and troughs, although the velocity of fluid motion in troughs is lower than in crests. This is because when the value of $\mathrm{N}_{\mathrm{R}}$ increases, the thermal radiation heat flux is absorbed into the fluid. Although the temperature of the fluid increases with increasing $\mathrm{N}_{\mathrm{R}}$, phase change does not take place in the micro-polar fluid, hence the value of total fluid viscosity remains constant. The y axial distribution of micro-rotation $\mathrm{N}$ is shown in Figure 10. The micro-rotation gradient of $\mathrm{N}$ along the $\mathrm{y}$ direction is positive when $\mathrm{y}>2$. However, close to the wavy surface, the micro-rotation gradient of $\mathrm{N}$ along the $\mathrm{y}$ direction is negative. In other words, a negative micro-rotation gradient trends to retard the velocity of fluid motion near the plate, but a positive micro-rotation gradient accelerates the velocity of fluid motion away from the wavy surface, hence the velocity of fluid motion in a trough is lower than at a crest. 
Figure 8. The temperature profiles of $\theta$ for different $N_{R}$ while $\alpha=0.004, \operatorname{Pr}=0.7$, $\mathrm{Br} / \Omega=1, \theta_{w}=1.2$.

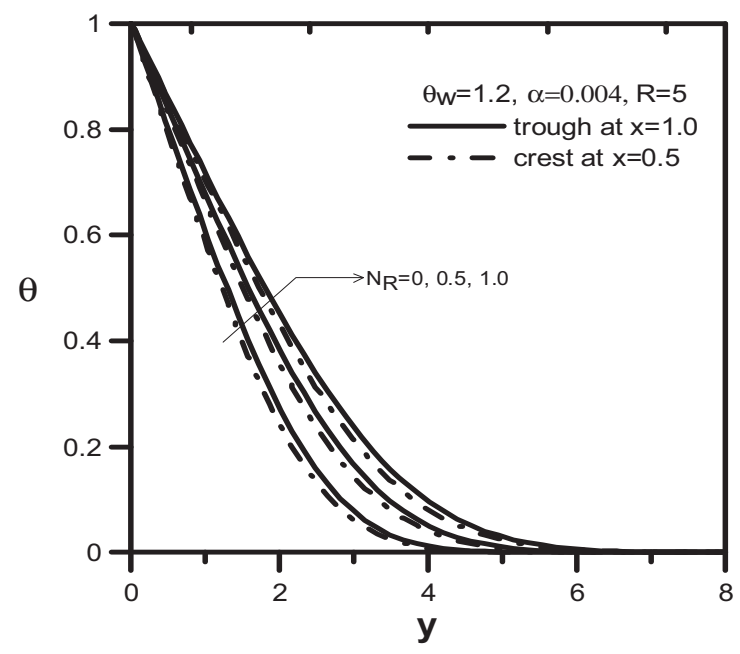

Figure 9. The axial velocity profiles of $u$ for different $N_{R}$ while $\alpha=0.004, \operatorname{Pr}=0.7$, $\operatorname{Br} / \Omega=1, \theta_{w}=1.2$.

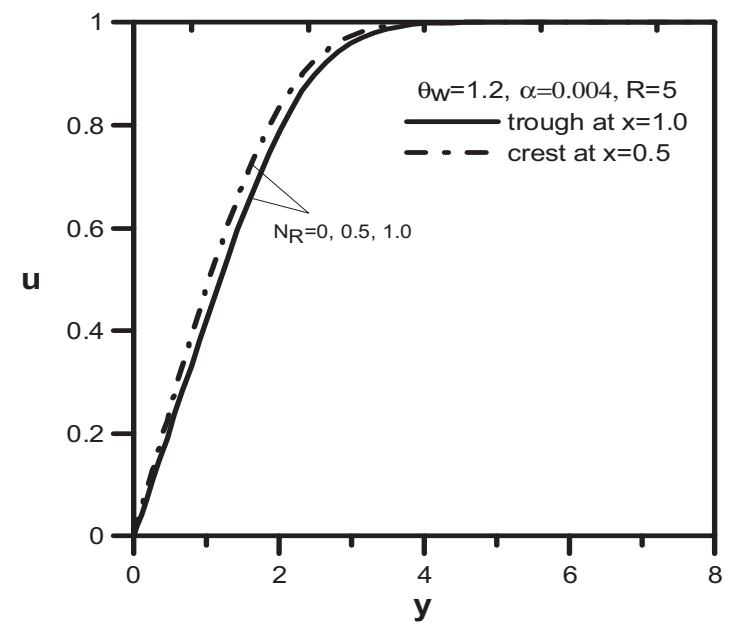

Figure 10. The micro-rotation profiles of $N$ for different $N_{R}$ while $\alpha=0.004, \operatorname{Pr}=0.7$, $\mathrm{Br} / \Omega=1, \theta_{w}=1.2$.

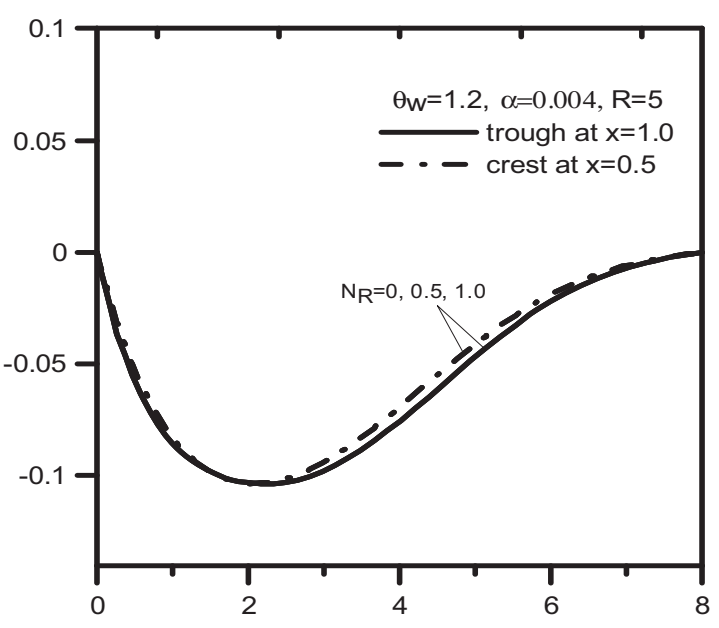




\section{Conclusions}

In this paper, the effect of thermal radiation on micro-polar fluid flow over a wavy surface has been studied. A modified form of the entropy generation equation has been derived. Effects of thermal radiation on the temperature and the vortex viscosity parameter, and the effect of the wavy surface on the velocity are all included in the modified entropy generation equation. The general results of this investigation are summarized as follows. When the value of $\mathrm{R}$ remains constant, then $\mathrm{N}_{\mathrm{R}}$ has no effect on the relation between $\mathrm{R}$ and $\mathrm{N}_{\mathrm{F}}$. That is because the values of $\mathrm{N}_{\mathrm{R}}$ and $R$ are not coupled, and the value of $B r / \Omega$ is constant. In other words, with increasing value of $N_{R}$, the thermal radiation heat flux is absorbed into the fluid. Although the temperature of the fluid increases with increasing $\mathrm{N}_{R}$, phase change does not take place in the micro-polar fluid, so the value of fluid total viscosity remains constant and entropy generation due to fluid friction is maintained at a constant level. From the results and discussion, moreover, it is seen that when $\mathrm{N}_{\mathrm{R}}$ increases, the thermal radiation heat flux is absorbed into the fluid, which leads to enhanced entropy generation due to heat transfer of the flow field, so heat transfer dominates entropy generation for the wavy surface. In addition, it is seen that a negative micro-rotation gradient trends to retard the fluid velocity near the plate, but a positive micro-rotation gradient accelerates the velocity of fluid motion away from the wavy surface, hence the fluid velocity in the troughs is lower than at the crests.

\section{Acknowledgments}

Thanks for the assistance from National Science Council (96-2221-E-006-171-MY2).

\section{References}

1. Yao, L.S. Natural convection along a vertical wavy surface. ASME J. Heat Trans. 1983, 105, 465-468.

2. Yao, L.S. A note on Prandtl's transposition theorem. ASME J. Heat Trans. 1988, 100, 507-508.

3. Moulic, S.G.; Yao, L.S. Natural convection along a vertical wavy surface with uniform heat flux. ASME J. Heat Trans. 1989, 111, 1106-1108.

4. Yao, L.S. Natural convection along a vertical complex wavy surface. Int. J. Heat Mass Trans. 2006, 49, 281-286.

5. Wang, C.C.; Chen, C.K. Forced convection in a wavy-wall channel. Int. J. Heat Mass Trans. 2002, $45,2587-2595$.

6. Ariman, T.; Turk, M.A.; Sylvester, N.D. Microcontinuum fluid mechanics, a review. Int. J. Eng. Sci. 1973, 11, 905-930.

7. Ariman, T.; Turk, M.A.; Sylvester, N.D. Applications of microcontinuum fluid mechanics. Int. J. Eng. Sci. 1974, 12, 273-293.

8. Eringen, A.C. Theory of micropolar fluids. J. Math. Mech. 1966, 16, 1-18.

9. Eringen, A.C. Theory of thermomicro fluids. J. Math. Anal. Appl. 1972, 38, 480-496.

10. Wang, C.C.; Chen, C.K. Forced convection in micropolar fluid flow over a wavy surface. Numer. Heat Trans. 2000, 37, 271-279. 
11. Wang, C.C.; Chen, C.K. Transient force and free convection along a vertical wavy surface in micropolar fluids. Int. J. Heat Mass Trans. 2001, 44, 3241-3251.

12. Lien, F.S.; Chen, T.M.; Chen, C.K. Analysis of a free-convection micropolar boundary layer about a horizontal permeable cylinder at a non-uniform thermal condition. ASME J. Heat Trans. 1990, 112, 504-506.

13. Lien, F.S.; Chen, C.K.; Cleaver, J.W. Analysis of natural convection flow of micropolar fluid about a sphere with blowing and suction. ASME J. Heat Trans. 1986, 108, 967-970.

14. Yang, Y.T.; Chen, C.K.; Lin, M.T. Natural convection of non-Newtonian fluids along a wavy vertical plate including the magnetic field effect. Int. J. Heat Mass Trans. 1996, 39, 2831-2842.

15. Chen, C.K.; Yang, Y.T.; Lin M.T. Transient free convection of non-Newtonian fluids along a wavy vertical plate including the magnetic field effect. Int. J. Heat Fluid Flow 1996, 17, 604-612.

16. Wang, C.C.; Chen, C.K. Mixed convection boundary layer flow of non-Newtonian fluids along vertical wavys plates. Int. J. Heat Fluid Flow 2002, 23, 831-839.

17. Lien, F.S.; Chen, C.K.; Cleaver, J.W. Radiation effect on conjugated forced convection-conduction analysis of heat Trans. in a plate fin. AIAA J. 1985, 23, 1645-1647.

18. Rosseland, S. Theoretical Astrophysics; Oxford University Press: London, UK, 1936.

19. Novotny, J.L.; Yang, K.T. The interaction of thermal radiation in optically thick boundary layers. ASME J. Heat Trans. 1967, 192, 309-312.

20. Cheng E.H. Ozisic M.N. Radiation with free convection in an absorbing, emitting and scattering medium. Int. J. Heat Mass Trans. 1972, 15, 1243-1252.

21. Hossain, M.A. Takhar, H.S. Radiation effect on mixed convection along a vertical plate with uniform surface temperature. Heat Mass Trans. 1996, 31, 243-248.

22. Elsayed, M.M.; Fathalah, K.A. Natural convection over a vertical fiat plate due to absorption of thermal radiation. Heat Mass Trans. 1985, 19, 121-129.

23. Hooman, K.; Hooman, F.; Mohebpour, S.R. Entropy generation for forced convection in a porous channel with isoflux or isothermal walls. Int. J. Exer. 2008, 5, 78-96.

24. Makinde, O.D. Entropy-generation analysis for variable-viscosity channel flow with non-uniform wall temperature. Appl. Energ. 2008, 85, 384-393.

25. Makinde, O.D.; Anwar, B.O. On inherent irreversibility in a reactive hydromagnetic channel flow. J. Therm. Sci. 2010, 19, 72-79.

26. Erbay, L.B.M.; Yalçın, M.M.; Ercan, M.S. Entropy generation in parallel plate microchannels. Heat Mass Trans. 2007, 43, 729-739.

27. Makinde, O.D. Thermodynamic second law analysis for a gravity driven variable viscosity liquid film along an inclined heated plate with convective cooling. J. Mech. Sci. Technol. 2010 24, 899-908.

28. Paoletti, S.; Rispoli, F.; Sciubba, E. Calculation of exergetic losses in compact heat exchanger passages. ASME AES 1989, 10, 21-29.

29. Antar, M.A.; El-Shaarawi, M.A.I. Entropy generation due to forced convection fluid flow about a solid sphere. Int. J. Exer. 2008, 5, 97-114.

30. Chen, C.K.; Su, Y.F. Exergetic efficiency optimization for an irreversible Brayton refrigeration cycle. Int. J. Therm. Sci. 2005, 44, 303-310. 
31. Cheng, C.Y.; Chen, C.K. Efficiency optimizations of an irreversible Brayton heat engine. Trans. ASME J. Energ. Resour. Technol. 1998, 120, 143-148.

32. Su, Y.F.; Chen, C.K. Analysis and optimisation of an irreversible Brayton refrigeration cycle using exergy concept. Int. J. Exer. 2008, 5, 470-486.

33. Erbay, L.B.; Göktun S.; Yavuz, H. Optimal design of the regenerative gas turbine engine with isothermal heat addition. Int. J. Appl. Energ. 2001, 68, 249-264.

34. Aziz, A.; Makinde, O.D. Entropy generation minimization design of a two-dimensional orthotropic convection Pin Fin. Int. J. Exer. 2010, 7, 579-592.

35. Hsu, P.T.; Chen, C.K.; Wang, C.C. Mixed convection of micropolar fluids along a vertical wavy surface. Acta Mech. 2000, 144, 231-247.

36. Moulic, S.G.; Yao, L.S. Mixed convection along a vertical wavy surface. ASME J. Heat Trans. 1989, 111, 974-978.

37. Bejan, A. A study of entropy generation in fundamental convective heat Transfer. ASME J. Heat Trans. 1979, 101, 718-725.

38. Bejan, A. Entropy Generation through Heat and Fluid Flow; Wiley: New York, NY, USA, 1982.

39. Mahmud, S.; Fraser, R.A. The second law analysis in fundamental convective heat Trans. problems. Int. J. Therm. Sci. 2003, 42, 177-186.

40. Rubin, S.G.; Graves, R.A. Viscous flow solution with a cubic Spline approximation. Comput. Fluid. 1975, 1, 1-36.

41. Wang, P.; Kahawita, R. Numerical integration of partial differential equations using cubic spline. Int. J. Comput. Math. 1983, 13, 271-286.

42. Çengel, Y.A. Heat and Mass Transfer: A Practical Approach; McGraw-Hill: New York, NY, USA, 2004.

43. Chen, C.K.; Yang, Y.T.; Chang, K.H. Entropy generation of laminar-forced convection along the wavy surface. Int. J. Exer. 2010, 7, 564-578.

(C) 2011 by the authors; licensee MDPI, Basel, Switzerland. This article is an open access article distributed under the terms and conditions of the Creative Commons Attribution license (http://creativecommons.org/licenses/by/3.0/). 